\title{
Injection of $\mathrm{CD} 86^{+}$macrophages instead of liver partition for the acceleration of liver regeneration after portal vein ligation in rats
}

\author{
Jinwei Zhao' ${ }^{1}$ Weiyi Zhao ${ }^{2}$, Hong Zhou ${ }^{1}$, Hongyue Xu ${ }^{1}$, Lu Yu
}

\begin{abstract}
${ }^{1}$ Department of Hepatopancreatobiliary Surgery of Second Hospital of Jilin University, Key Laboratory for Zoonosis Research, Ministry of Education, Institute of Zoonosis, College of Veterinary Medicine Jilin University, Changchun, China

${ }^{2}$ Medical College of YanBian University, YanBian, China
\end{abstract}

Submitted: 6 January 2021; Accepted: 15 March 2021;

Online publication: 21 March 2021

Arch Med Sci 2021; 17 (3): 843-847

DOI: https://doi.org/10.5114/aoms/133361

Copyright $\odot 2021$ Termedia \& Banach

Partial hepatectomy $(\mathrm{PH})$ remains the first-line curative treatment for liver malignancies; however, an extended hepatectomy is accompanied by higher risk of postoperative liver failure, which is the most common cause of mortality after this procedure. Whether a hepatic resection is performed generally depends on preoperative liver function and the volume of the further liver remnant (FLR) [1-4].

Previous studies have demonstrated that Kupffer cells produce important cytokines that induce cell proliferation after hepatectomy [3]. For the present study, it was hypothesized that, instead of performing a liver parenchymal transection after portal vein ligation (PVL), injecting polarized proinflammatory $\mathrm{CD} 86^{+}$macrophages could trigger a full inflammatory response and accelerate liver regeneration through the release of proinflammatory cytokines.

Inbred male Sprague-Dawley (SD) rats aged 8-10 weeks (body weight 260-290 g) were obtained from the Animal Laboratories of Jilin University, Changchun, China. The study was in compliance with the Guide for the Care and Use of Laboratory Animals published by the US National Institutes of Health (NIH publication no. 85-23, revised 1996).

The rats were separated into four groups: (1) a sham group, in which the abdomen was opened and closed after manipulation of the liver hilum $(n=6)$; (2) a PVL group, in which a caudate lobectomy and selective ligation of the portal vein feeding the left median, left lateral, and right lobes were performed $(n=6)$; (3) an associating liver partition and portal vein ligation for staged hepatectomy (ALPPS) group, in which a caudate lobectomy and a parenchymal transection between the right and left median lobes after selective PVL were performed $(n=6)$; (4) a PVL/injection of $\mathrm{CD} 86^{+}$macrophages group (PVL/macrophages), in which $\mathrm{CD} 86^{+} \mathrm{mac}$ rophages were injected into the body via the portal vein after the PVL procedure $(n=6)$. Four time points were used during the experiment: postoperative day 1 (POD1), postoperative day 3 (POD3), postoperative day 5 (POD5), and postoperative day 7 (POD7).

Macrophages were recovered from the peritoneal cavity of euthanized $S D$ rats using $10 \mathrm{ml}$ of ice-cold phosphate buffer saline (PBS) supplemented with ethylenediaminetetraacetic acid (EDTA). Purified macrophages were incubated for $24 \mathrm{~h}$ without fetal bovine serum (FBS) and with $20 \mathrm{ng} / \mathrm{ml}$ of interferon gamma (IFN- $\gamma$ ), before being stimulated with

\author{
Corresponding author: \\ Lu Yu \\ Department of \\ Hepatopancreatobiliary \\ Surgery \\ Second Hospital \\ of Jilin University \\ Key Laboratory for \\ Zoonosis Research \\ Ministry of Education \\ Institute of Zoonosis \\ College of Veterinary \\ Medicine Jilin University \\ 218 Ziqiang St \\ Nanguan District \\ Changchun 130041 \\ China \\ Phone: +86 0431-88796222 \\ E-mail: yulucm447@163.com
}


Table I. Expression of M1 macrophage and levels of pro-inflammatory cytokines in culture supernatant

\begin{tabular}{|lcc|}
\hline Parameter & $\begin{array}{c}\text { CD68 } \\
\text { macrophage } \\
\text { group }\end{array}$ & $\begin{array}{c}\text { CD86 }{ }^{+C D 68}{ }^{+} \\
\text {macrophage } \\
\text { group }\end{array}$ \\
\hline Cell purity(\%) & $96.0 \pm 0.9$ & $98.1 \pm 0.7$ \\
\hline Cytokines[pg/ml]: & $601.4 \pm 62.1$ & $1165.0 \pm 76.4^{*}$ \\
\hline IL-2 & $70.9 \pm 5.2$ & $181.6 \pm 18.5^{*}$ \\
\hline IL-6 & $105.5 \pm 5.8$ & $155.8 \pm 7.8^{*}$ \\
\hline TNF- $\alpha$ & $14.2 \pm 1.4$ & $32.6 \pm 4.7^{*}$ \\
\hline IL-1 $\beta$ &
\end{tabular}

Values are means $\pm S D .{ }^{*} p<0.01$. IL-2-interleukin-2, IL-6-interleukin-6, TNF- $\alpha$ - tumor necrosis factor $\alpha, I L-2 \beta$ - interleukin- $\beta$.

$500 \mathrm{ng} / \mathrm{ml}$ of lipopolysaccharide (LPS). The cells were then stained using indicated fluorochrome-conjugated antibodies for $30 \mathrm{~min}$ at $4^{\circ} \mathrm{C}$ before being washed with PBS supplemented with $2 \%$ bovine serum albumin (BSA), $4 \mathrm{mM}$ EDTA, and $0.01 \%$ sodium azide $\left(\mathrm{NaN}_{3}\right)$ and fixed in $4 \%$ paraformaldehyde.

The culture supernatant of the macrophages was collected $24 \mathrm{~h}$ after being treated with $20 \mathrm{ng} / \mathrm{ml}$ IFN- $\gamma$ and $500 \mathrm{ng} / \mathrm{ml}$ LPS. Tumor necrosis factor $\alpha$ (TNF- $\alpha$ ), interleukin-6 (IL-6), interleukin-1 $\beta$ (IL-1 $\beta$ ), and interleukin-2 (IL-2) in the cell culture supernatant were measured using enzyme-linked immunosorbent assay (ELISA) kits according to the manufacturer's instructions (ABclonal Biotechnology Co. Ltd., Wuhan, China).

The rats were anesthetized with $30 \mathrm{mg} / \mathrm{kg}$ barbital sodium (Ketalar; Par Pharmaceutical Co. Inc., Spring Valley, New York, USA) via intraperitoneal injection after the skin was cleaned with iodine and $70 \%$ alcohol. All surgical procedures were performed under an operating microscope (binocular operation microscope, type GX.SS.22-3; Shanghai Medical Optical Instruments Co. Ltd., China). Transection of the liver parenchyma was carried out using bipolar coagulation forceps (electrosurgery unit, ART-E1; Bonart Co. Ltd., Taipei, Taiwan).

The HRR of the right median lobe was calculated using the formula $\mathrm{HRR}=\left(W_{A}-W_{l}\right) / W_{1} \times 100 \%$ (5), where $W_{A}$ was the actual weight of the right median lobe measured at the time of death and $W$, was the initial weight of the lobe before surgery. The initial weight of the right median lobe was calculated with the formula $W_{1}=$ body weight $\times 0.97 \%$. The value of $0.97 \%$ represented the mean right median lobe weight as a percentage of the body weight of 10 normal male SD rats weighing 260-290 g.

Liver tissue was stained with hematoxylin and eosin (H\&E). The numbers of $\mathrm{Ki}-67^{+}$hepatocytes and $\mathrm{CD} 86^{+}$macrophages were counted using an immunohistochemical staining assay with the $\mathrm{Ki}-67$ and $\mathrm{CD}^{2} 6^{+}$antibodies according to the manufacturer's instructions (ABclonal Biotechnology Co. Ltd., Wuhan, China).
All animal experiments were carried out according to the experimental practices and standards approved by the Animal Welfare and Research Ethics Committee at Jilin University (no: IZ-2009008). The experimental protocols were reviewed and approved by the committee.

Data were expressed as mean \pm standard deviation (SD). Differences between the groups were assessed using the Mann-Whitney $U$ test and Student's t-test. Statistical analyses were conducted using Prism 5.0 (GraphPad Software Inc, San Diego California, USA). $P<0.05$ was considered statistically significant.

The injection of polarized macrophages resulted in significantly increased production of $\mathrm{IL}-2$, IL-6, TNF- $\alpha$, and IL- $1 \beta$ compared with the injection of non-polarized macrophages $(p<0.01)$ (Table I).

Compared with the PVL group, the ALPPS group and $\mathrm{PVL} / \mathrm{macrophage}$ group had a greater regeneration response, with an increased HRR at POD3, POD5, and POD7 $(p<0.01)$. Although the HRR of the ALPPS group was higher than that of the PVL/ macrophage group at POD3 $(p<0.01)$, there was a gradual increase in the HRR of the PVL/macrophage group. At POD5, the increased HRR of the $\mathrm{PVL} / \mathrm{macrophage}$ group was comparable to the HRR of the ALPPS group; however, the increased HRR was significantly lower in the PVL/macrophage group than in the ALPPS group at POD7 $(p<0.05)$.

There was a significant difference between the number of $\mathrm{CD}^{+} 6^{+}$macrophages per visual field (magnification 20x) in the four groups at POD1, POD3, and POD5. The number of $\mathrm{CD}^{2} 6^{+}$macrophages was significantly higher in the ALPPS group and the PVL/macrophage group than in the PVL and sham groups at POD1, POD3, and POD5 $(p<0.01)$, while the number of $\mathrm{CD} 86^{+}$macrophages was significantly higher in the ALPPS group than in the PVL/ macrophage group $(p<0.05)$ (Figures $1 \mathrm{~A}, \mathrm{~B})$.

The serum levels of IL- 6 and IL- $1 \beta$ were significantly elevated in the ALPPS and PVL/macrophage groups at POD1 and POD3 compared with those of the PVL group $(p<0.01)$ at the same time point. There were significant differences in levels of serum TNF- $\alpha$ (PVL group vs. ALPPS group) and IL-2 (PVL group vs. PVL/macrophage group) at POD1 $(p<0.01)$ and in the serum levels of IL-2 (PVL group vs. ALPPS group) and TNF- $\alpha$ (PVL group vs. $\mathrm{PVL} /$ macrophage group) at POD3 ( $p<0.01)$. It was observed that the serum HGF levels of the three surgical groups were significantly higher than those of the sham group $(p<0.01)$, and there were no differences between the HGF levels of the surgical groups at any of the time points except in the ALPPS group, which had higher levels than the $\mathrm{PVL} / \mathrm{macrophage}$ group at POD3 $(p<0.01)$ (Table II, Figures $1 \mathrm{C}-\mathrm{G}$ ).

In this rat surgical model, although both the PVL and ALPPS groups exhibited accelerated liver 
A
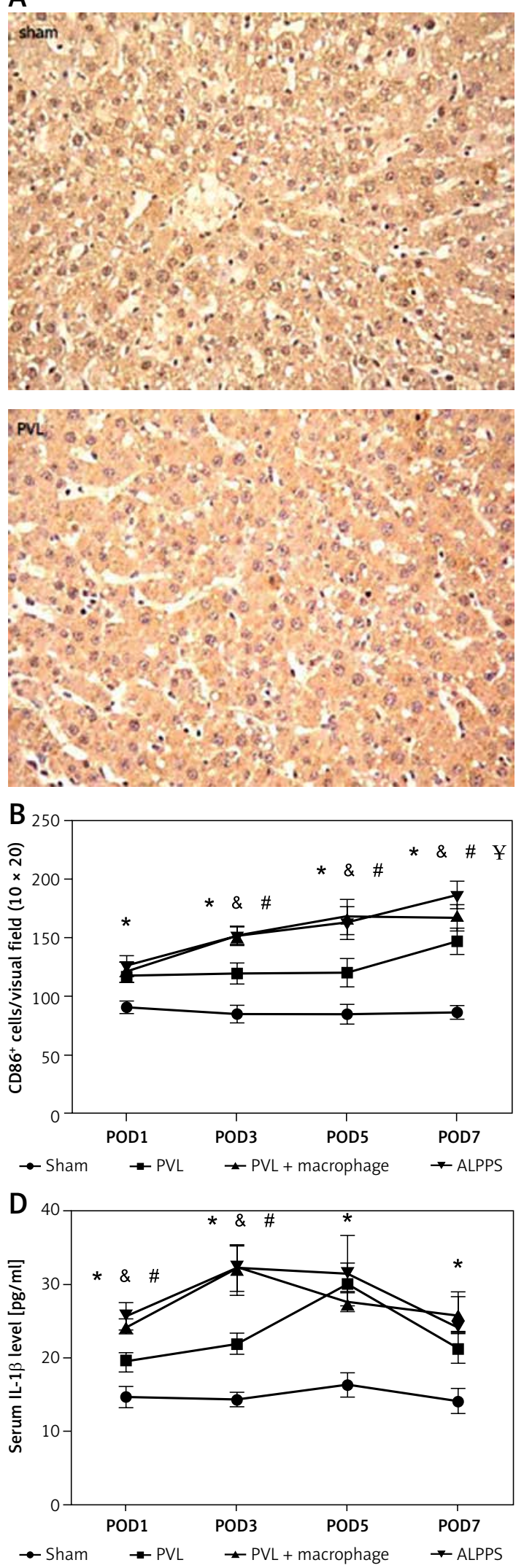
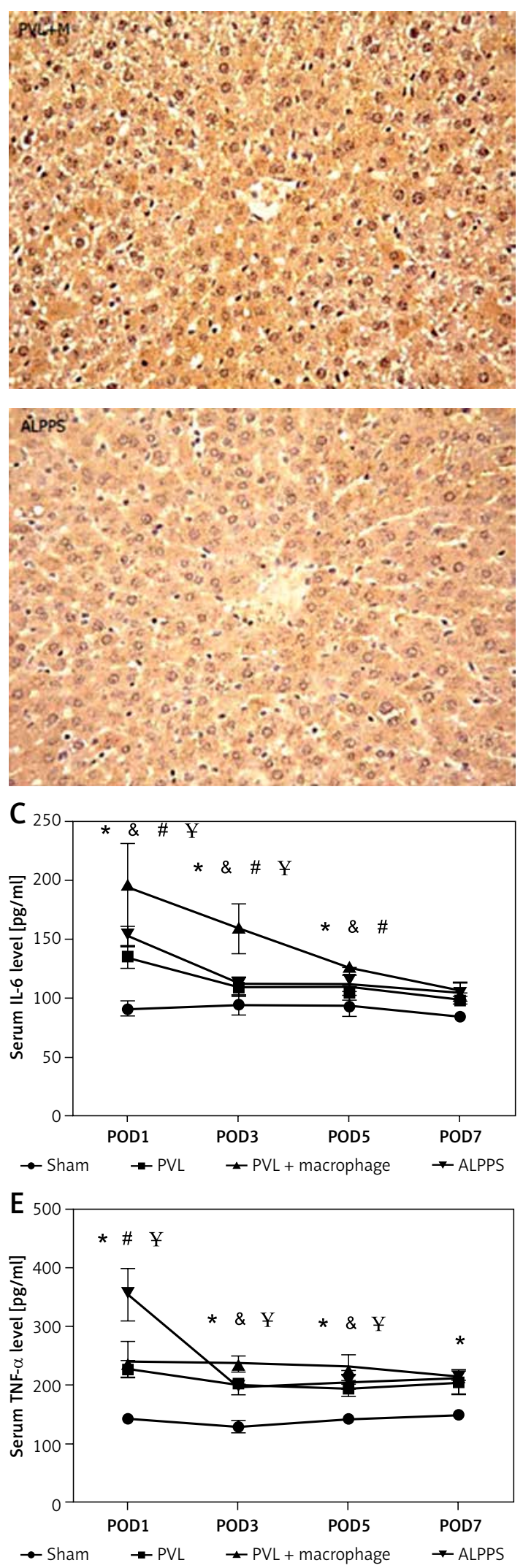

Figure 1. Infiltration of Kupffer cells and levels of proinflammatory cytokines and HGF. A - Immunohistochemical staining for $\mathrm{CD} 86^{+}$in regenerating lobes at POD7. Kupffer cells with nuclear deposition of blue pigment were positive (original magnification 200x). B - The number of CD86+ macrophages per visual field (magnification 20x). C - Serum IL-6 levels. D - Serum IL-1 $\beta$ levels. E - Serum TNF- $\alpha$ levels

Values are means $\pm S D$. ${ }^{*} p<0.01$ compared with the sham group; ${ }^{*} p<0.01$ PVL group vs. PVL/macrophage group; ${ }^{*} p<0.01 P V L$ group vs. ALPPS group; ${ }^{*} p<0.01 \mathrm{PVL} /$ macrophage group vs. ALPPS group. 
F

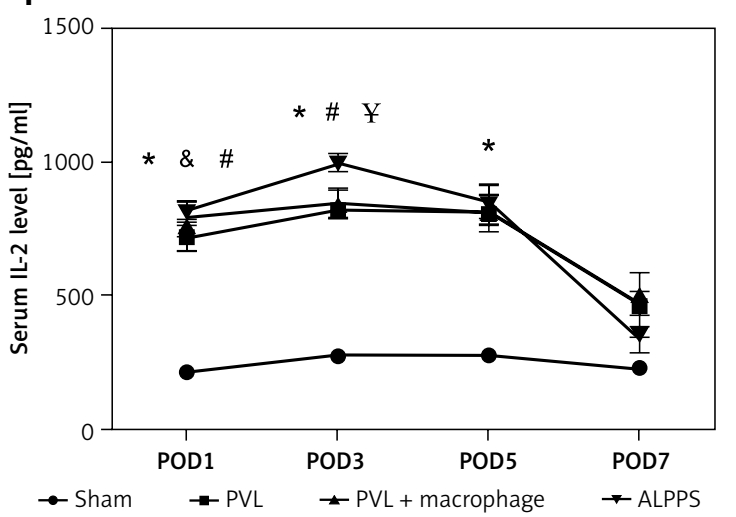

G

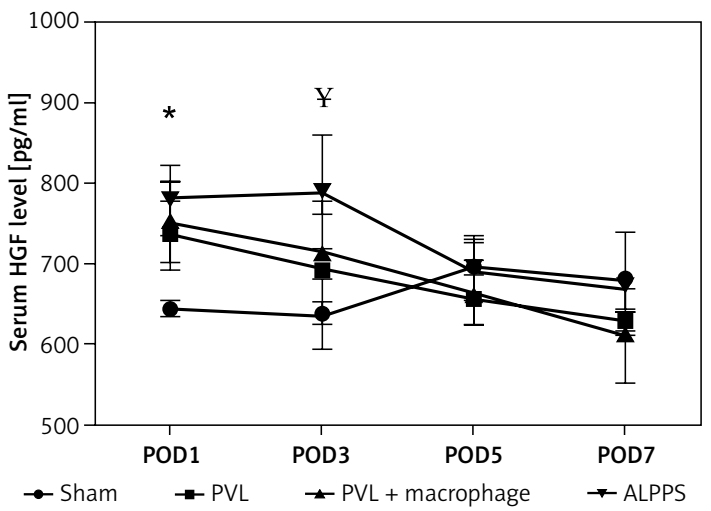

Figure 1. Cont. F - Serum IL-2 levels. G - Serum HGF levels

Values are means $\pm S D$. ${ }^{*} p<0.01$ compared with the sham group; ${ }^{*} p<0.01 P V L$ group $v s . P V L /$ macrophage group; ${ }^{*} p<0.01 P V L$ group vs. ALPPS group; ${ }^{*} p<0.01$ PVL/macrophage group vs. ALPPS group.

regeneration in the FLR compared with the sham group, ALPPS was more effective. The levels of IL- 6 , TNF- $\alpha$, IL-1 $\beta$, IL-2, and HGF were significantly elevated after ALPPS but not after PVL alone, suggesting that proinflammatory cytokines and HGF could play a vital role in accelerating liver regeneration after ALPPS. Previous studies using the rodent model of ALPPS strongly suggest that parenchymal transection induces an inflammatory response and releases proliferating factors, which in turn induces accelerated regeneration of the FLR [5-7]. To assess the effect of this accelerated regeneration, an injection of proinflammatory $\mathrm{CD}^{2} 6^{+}$macrophages was administered during the PVL procedure in the present study. It was found that the HRR of the PVL/macrophage group was higher than that of the PVL group at POD3, POD5, and POD7. In addition, the HRR in the PVL/macrophage group was similar to that of the ALPPS group at POD5. These results suggest that injec-

Table II. Protein levels of proinflammatory cytokines and HGF

\begin{tabular}{|c|c|c|c|c|c|}
\hline Variable & IL-6 [pg/ml] & TNF- $\alpha[\mathrm{pg} / \mathrm{ml}]$ & $\mathrm{IL}-2$ [pg/ml] & $\mathrm{IL}-1 \beta[\mathrm{pg} / \mathrm{ml}]$ & $\mathrm{HGF}[\mathrm{pg} / \mathrm{ml}])$ \\
\hline \multicolumn{6}{|l|}{ Sham: } \\
\hline POD1 & $93.1 \pm 6.7^{\star}$ & $142.2 \pm 8.6^{*}$ & $216.1 \pm 12.9^{*}$ & $14.8 \pm 1.4^{\star}$ & $645.1 \pm 12.1^{*}$ \\
\hline POD3 & $92.2 \pm 8.3^{*}$ & $131.5 \pm 11.2^{*}$ & $280.3 \pm 26.5^{*}$ & $14.5 \pm 1.0^{\star}$ & $637.0 \pm 45.3$ \\
\hline POD5 & $94.4 \pm 8.8^{*}$ & $143.3 \pm 9.1^{*}$ & $281.3 \pm 23.2^{*}$ & $16.4 \pm 1.6^{\star}$ & $698.2 \pm 37.3$ \\
\hline POD7 & $85.1 \pm 4.3$ & $155.1 \pm 5.2^{*}$ & $232.1 \pm 22.0$ & $14.2 \pm 1.6^{\star}$ & $679.2 \pm 62.1$ \\
\hline \multicolumn{6}{|l|}{ PVL: } \\
\hline POD1 & $137.0 \pm 8.4^{\& \#}$ & $227.0 \pm 14.0^{\#}$ & $717.6 \pm 45.4^{8}$ & $19.5 \pm 1.4^{\& \#}$ & $743.0 \pm 45.1$ \\
\hline POD3 & $110.0 \pm 3.4^{\& \#}$ & $213.1 \pm 8.6^{8}$ & $818.6 \pm 20.9^{\#}$ & $22.0 \pm 1.5^{\& \#}$ & $688.3 \pm 67.6$ \\
\hline POD5 & $109.9 \pm 10.4^{8}$ & $194.8 \pm 12.4$ & $816.8 \pm 40.4$ & $30.2 \pm 2.9$ & $657.0 \pm 31.5$ \\
\hline POD7 & $98.4 \pm 4.1$ & $204.2 \pm 18.6$ & $476.6 \pm 43.4$ & $21.0 \pm 2.0$ & $628.8 \pm 14.9$ \\
\hline \multicolumn{6}{|c|}{ PVL + macrophage: } \\
\hline POD1 & $196.1 \pm 36.0^{*}$ & $243.9 \pm 30.9^{*}$ & $798.7 \pm 41.0$ & $24.4 \pm 1.1$ & $753.5 \pm 50.2$ \\
\hline POD3 & $159.9 \pm 20.7^{\ngtr}$ & $238.3 \pm 11.9^{*}$ & $848.9 \pm 50.0^{*}$ & $32.1 \pm 3.3$ & $714.4 \pm 62.4^{\xi}$ \\
\hline POD5 & $126.5 \pm 4.6^{*}$ & $231.7 \pm 20.7^{*}$ & $810.0 \pm 67.1$ & $27.9 \pm 1.2$ & $664.3 \pm 40.0$ \\
\hline POD7 & $108.3 \pm 5.5$ & $212.2 \pm 8.1$ & $463.7 \pm 119.0$ & $26.0 \pm 2.4$ & $612.0 \pm 58.1$ \\
\hline \multicolumn{6}{|l|}{ ALPPS: } \\
\hline POD1 & $153.9 \pm 8.7$ & $353.1 \pm 43.4$ & $817.9 \pm 35.3$ & $25.8 \pm 1.8$ & $788.0 \pm 48.7$ \\
\hline POD3 & $115.4 \pm 4.2$ & $196.9 \pm 11.1$ & $998.3 \pm 30.8$ & $32.4 \pm 3.1$ & $798.9 \pm 21.4$ \\
\hline POD5 & $112.4 \pm 4.7$ & $204.6 \pm 12.2$ & $852.0 \pm 64.8$ & $31.6 \pm 5.2$ & $690.9 \pm 36.3$ \\
\hline POD7 & $105.9 \pm 3.8$ & $223.4 \pm 6.9$ & $339.7 \pm 44.8$ & $24.3 \pm 4.8$ & $677.1 \pm 5.2$ \\
\hline
\end{tabular}

Values are means $\pm S D ;{ }^{*} p<0.01$ compared with the sham group; ${ }^{*} p<0.01 P V L$ group vs. $P V L+$ macrophage group; ${ }^{*} p<0.01 P V L$ group vs. ALPPS group; ${ }^{*} p<0.01 P V L+$ macrophage group vs. ALPPS group; ${ }^{\ddagger} p<0.01 P V L+$ macrophage group VS. ALPPS group. PVL - portal vein ligation, $P O D 1$ - postoperative day $1, P O D 3$ - postoperative day 3, POD5 - postoperative day 5, POD7 - postoperative day 7, IL-2 interleukin-2, IL-6 - interleukin-6, TNF- $\alpha$ - tumor necrosis factor $\alpha, I L-1 \beta$ - interleukin-1 $\beta$, HGF - hepatocyte growth factor. 
tion of proinflammatory $\mathrm{CD} 86^{+}$macrophages after PVL accelerates liver regeneration. To further explore whether the PVL/proinflammatory CD86 ${ }^{+}$ macrophage procedure could have the same mechanisms as the ALPPS procedure in accelerating liver regeneration, the study measured the levels of IL-6, TNF- $\alpha$, IL-1 $1 \beta$, IL-2 and HGF in serum samples and $\mathrm{CD} 86^{+}$macrophages in the FLR.

In conclusion, proinflammatory CD86 $6^{+}$macrophages combined with PVL can induce accelerated liver regeneration comparable to the FLR growth seen after ALPPS. Proinflammatory cytokines secreted by $\mathrm{CD}^{2} 6^{+}$macrophages and the increased number of $\mathrm{CD}^{+} 6^{+}$macrophages in the FLR may play a role in the acceleration of FLR regeneration.

\section{Acknowledgments}

This work was supported by the Fund for Science \& Technology Development of Jilin Province (20160101060JC, 20200201544JC); the National Key R\&D Program of China (2017YFD0502200, 2016YFD0501302); the Project of the Education Department of Jilin Province (2016444).

\section{Conflict of interest}

The authors declare no conflict of interest.

\section{References}

1. Björnsson B, Sparrelid E, Røsok B, et al. Associating liver partition and portal vein ligation for staged hepatectomy in patients with colorectal liver metastases - intermediate oncological results. Eur J Surg Oncol 2016; 42: 531-7.

2. Schadde E, Ardiles V, Robles-Campos R, et al. Early survival and safety of ALPPS: first report of the International ALPPS Registry. Ann Surg 2014; 260: 829-38.

3. Zhan C, Lin G, Huang Y, Wang Z, Zeng F, Wu S. A dopamine-precursor-based nanoprodrug for in-situ drug release and treatment of acute liver failure by inhibiting NLRP3 inflammasome and facilitating liver regeneration. Biomaterials 2021; 268: 120573.

4. Zhang GQ, Zhang ZW, Lau WY, Chen XP. Associating liver partition and portal vein ligation for staged hepatectomy (ALPPS): a new strategy to increase resectability in liver surgery. Int I Surg 2014; 12: 437-41.

5. Yao L, Li C, Ge X, et al. Establishment of a rat model of portal vein ligation combined with in situ splitting. PLoS One 2014; 9: e105511.

6. Schlegel A, Lesurtel M, Melloul E, et al. ALPPS: from human to mice highlighting accelerated and novel mechanisms of liver regeneration. Ann Surg 2014; 260: 839-47.

7. Shi H, Yang G, Zheng T, et al. A preliminary study of ALPPS procedure in a rat model. Sci Rep 2015; 5: 17567. 